INPLASY

PROTOCOL

To cite: Wu et al. Efficacy of Endoscopic Lumbar Interbody Fusion (Endo-LIF) for Lumbar Spinal Stenosis: A Systematic Review and Meta-Analysis. Inplasy protocol 2021120097. doi:

10.37766/inplasy2021.12.0097

Received: 22 December 2021

Published: 22 December 2021

Corresponding author:

Wu Ruiqing

1215657821@qq.com

Author Affiliation:

Jiangxi University of Chinese Medicine.

Support: Horizontal Subject.

Review Stage at time of this submission: Preliminary

searches.

Conflicts of interest:

None declared.

\section{Efficacy of Endoscopic Lumbar Interbody Fusion (Endo-LIF) for Lumbar Spinal Stenosis: A Systematic Review and Meta-Analysis}

$\mathrm{Wu}, \mathrm{R}^{1} ; \mathrm{Ye}, \mathrm{Y}^{2}$.

Review question / Objective: P? Patients with Lumbar Spinal Stenosis. I? Endoscopic lumbar interbody fusion. C? Other lumbar interbody fusion. O?Predefined outcome measures were preoperative and postoperative visual analogue scale back and/or leg pain (VAS-BP) and Oswestry Disability Index (ODI) score; operation time; intraoperative blood loss; length of hospital stay; and the complications, reoperation and fusion rate. S: randomized controlled trials (RCTs) or nonrandomized cohort studies.

Condition being studied: Endoscopic Lumbar Interbody Fusion can be widely used for the treatment of lumbar spinal stenosis, and this study aims to summarize the efficacy of this procedure for lumbar spinal stenosis.Endoscopic Lumbar Interbody Fusion (Endo-LIF) for the treatment of Lumbar Spinal Stenosis.

INPLASY registration number: This protocol was registered with the International Platform of Registered Systematic Review and Meta-Analysis Protocols (INPLASY) on 22 December 2021 and was last updated on 22 December 2021 (registration number INPLASY2021120097).

\section{INTRODUCTION}

Review question / Objective: P? Patients with Lumbar Spinal Stenosis. I? Endoscopic lumbar interbody fusion. C? Other lumbar interbody fusion. 0 ? Predefined outcome measures were preoperative and postoperative visual analogue scale back and/or leg pain (VAS-
BP) and Oswestry Disability Index (ODI) score; operation time; intraoperative blood loss; length of hospital stay; and the complications, reoperation and fusion rate. S: randomized controlled trials (RCTs) or nonrandomized cohort studies.

Condition being studied: Endoscopic Lumbar Interbody Fusion can be widely 
used for the treatment of lumbar spinal stenosis, and this study aims to summarize the efficacy of this procedure for lumbar spinal stenosis.Endoscopic Lumbar Interbody Fusion (Endo-LIF) for the treatment of Lumbar Spinal Stenosis.

\section{METHODS}

Participant or population: Patients with Lumbar Spinal Stenosis.

Intervention: Endoscopic lumbar interbody fusion.

Comparator: Other lumbar interbody fusion.

Study designs to be included: Predefined outcome measures were preoperative and postoperative visual analogue scale back and/orleg pain (VAS-BP) and Oswestry Disability Index (ODI) score;operation time; intraoperative blood loss;lengthof hospital stay; and the complications, reoperation and fusion rate.

Eligibility criteria: Randomized controlled trials (RCTs) or nonrandomized cohort studies.

Information sources: PubMed, Embase, Cochrane, Web of Science.

Main outcome(s): Preoperative and postoperative visual analogue scale back and/or leg pain (VAS-BP) and Oswestry Disability Index (ODI) score.

Additional outcome(s): Operation time; intraoperative blood loss; length of hospital stay; and the complications, reoperation and fusion rate.

Quality assessment / Risk of bias analysis: Randomized controlled trials used the Cochrane Tool scale to evaluate the quality of the literature, and cohort studies used the NOS scale to evaluate the quality of the literature.

Strategy of data synthesis: If heterogeneity exists, choose random effects to combine the data, if not, choose fixed effects to combine the data.

Subgroup analysis: If necessary, subgroup analysis was performed in the surgical group to assess the complications of different procedures in the treatment of degenerative lumbar spine disease.

Sensitivity analysis: After deleting any of them, the combined results of the remaining papers are not significantly different from those when they are deleted, which means that the sensitivity analysis is passed.

Country(ies) involved: China.

Keywords: Spinal stenosis, Endoscopic Lumbar Interbody Fusion.

Contributions of each author:

Author 1 - Wu Ruiqing - Drafting of drafts, statistics and analysis of data, etc.

Email: 1215657821@qq.com

Author 2 - Ye Yongxiang - Literature search and data extraction, etc.

Email: 1194952548@qq.com 